\title{
Characterization of Microstrip Ring with a Narrow Gap by an Iterative Method
}

\author{
M. Yeddes, ${ }^{1}$ A. Gharsallah, ${ }^{1}$ A. Gharbi, ${ }^{1}$ and H. Baudrand ${ }^{2}$ \\ ${ }^{1}$ Laboratoire de Physique de la Matière Molle, Faculté des Sciences de Tunis, 2092 El Manar, Tunisia \\ ${ }^{2}$ Laboratoire d'Electronique, Groupe Microonde ENSEEIHT, 31071 Toulouse, France
}

Correspondence should be addressed to M. Yeddes, co_yeddes@voila.fr

Received 6 December 2006; Revised 12 April 2007; Accepted 27 February 2008

Recommended by Safleddin Safavi-Naeini

This paper presents an implementation of an iterative method based on the wave's concept, for analysing a ring resonator. This method includes a two-dimensional fourier modal transformation (2D-FMT) in a wave guide environment. The method has the advantage of simplicity in that it does not involve basis functions and inversion of matrices, as used in other calculation methods. Therefore, it is capable of analyzing larger bodies than other classical techniques. An implementation of the iterative calculation is shown for the extraction of $S$ parameters of microwave components. The good agreement between simulation results and experimental published data justifies the design procedure and validates the present analysis approach.

Copyright ( $) 2008$ M. Yeddes et al. This is an open access article distributed under the Creative Commons Attribution License, which permits unrestricted use, distribution, and reproduction in any medium, provided the original work is properly cited.

\section{Introduction}

In the Photonic Band Gap structures (PBG), an artificial lattice of dielectric can give rise to frequency regions where photons cannot propagate in any direction. Microwave circuits with a PBG structure are difficult to implement in compact form because such a structure requires at least five or six periods of units cells, and the size of one period is directly proportional to the wavelength of the center frequency of the PBG. In this paper, we show that a simple microstrip ring with a narrow gap can exhibit $\mathrm{PBG}$ characteristics.

An implementation of the iterative calculation is shown for extraction of $S_{i j}$ parameters of a ring resonator. It consists in generating a recursive relationship between a given wave source and reflected waves at the interface containing the circuit. This discontinuity plane is divided into cells and characterized by a scattering operator matrix depending on boundary conditions. Then, a 2D-FMT algorithm is used to pass from spatial to spectral domain for each iteration of the recursive process [1]. The regular ring shows $S$ parameters similar to those of a conventional transmission line; the introduction of a small gap in this regular ring induces two stop bands which can be tuned by a varactor incorporated into the narrow gap; this technique is detailed in the second part of this paper.

\section{Formulation}

The wave concept is introduced by writing the tangential electric field $E_{i}$ and surface tangential current density $J_{i}$ in terms of incident and reflected waves (see Figure 1) $[1,2]$. It leads to the following set of equations:

$$
\begin{array}{ll}
A_{i}=\frac{1}{2 \sqrt{Z_{o i}}} \quad\left(E_{i}+Z_{o i} J_{i}\right), \quad i=1,2, \\
B_{i}=\frac{1}{2 \sqrt{Z_{o i}}} \quad\left(E_{i}-Z_{o i} J_{i}\right), \quad i=1,2,
\end{array}
$$

where $\vec{J}_{i}$ is the surface tangential current density as $\vec{H}_{i} \wedge \vec{n}_{i}$, with $n_{i}$ a unit vector normal to the interface $\Omega ; A_{i}$ and $B_{i}$ are the incident and reflected waves associated with the discontinuity interface $\Omega ; Z_{0 i}$ is the characteristic impedance of the middle $i$, given by $Z_{0 i}=\sqrt{\mu_{0} / \varepsilon_{0} \varepsilon_{r i}}$, where $\varepsilon_{r i}$, is the relative permittivity of the region $I ; E_{i}$ and $H_{i}$ are the tangential electric field and magnetic field, respectively, on the plan $\Omega$. 


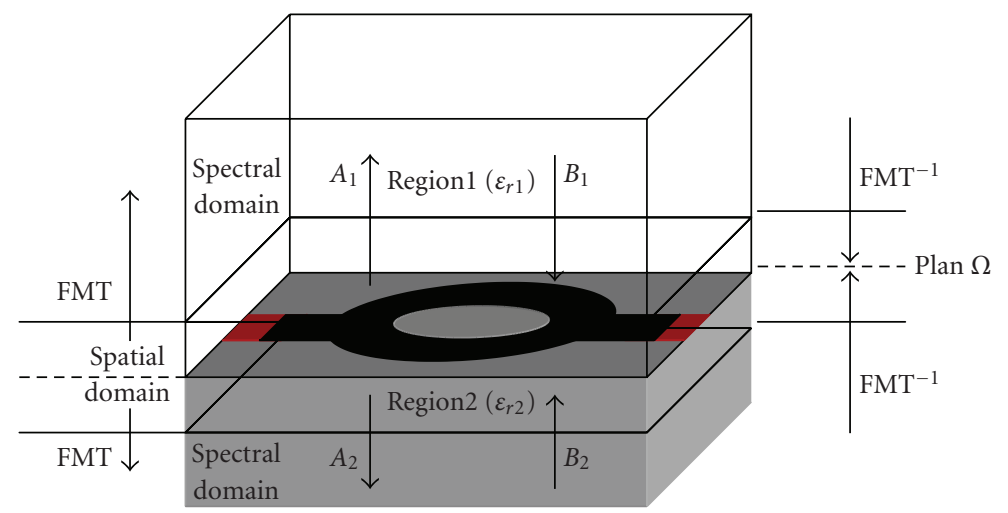

FIGURE 1: Definition of wave concept.

FMT: fourier modal transform.

On each region (region 1,2 ), it is possible to definite a scattering matrix on spatial domain and reflection coefficient on spectral domain.

The interface $\Omega$ (Spatial domain) in which the circuit is defined is divided into pixels [3]; the interface contains three subdomains (dielectric, metal, and source).

By using the boundary condition in each subdomain (dielectric, metal, and source), it is possible to define the scattering matrix $S_{M}$, who binds the incident waves to the reflected waves on the spatial domain $[1,3]$ :

$$
S_{M}=\left(\begin{array}{cc}
Q^{\prime} & Q^{\prime \prime} \\
Q^{\prime \prime \prime} & Q^{\prime \prime \prime \prime}
\end{array}\right)
$$

where $Q^{\prime}=-H_{m}-\left(\left(-1+n_{1}+n_{2}\right) /\left(1+n_{1}+n_{2}\right)\right) H_{s}+((1-$ $\left.\left.N^{2}\right) /\left(1+N^{2}\right)\right) H_{d}, Q^{\prime \prime}=\left(2 N /\left(1+N^{2}\right)\right) H_{d}+\left(2 n /\left(1+n_{1}+\right.\right.$ $\left.\left.n_{2}\right)\right) H_{s}, Q^{\prime \prime \prime}=\left(2 N /\left(1+N^{2}\right)\right) H_{d}+\left(2 n /\left(1+n_{1}+n_{2}\right)\right) H_{s}$, and $Q^{\prime \prime \prime \prime}=-H_{m}-\left(\left(-1-n_{1}+n_{2}\right) /\left(1+n_{1}+n_{2}\right)\right) H_{s}+\left(\left(1-N^{2}\right) /(1+\right.$ $\left.\left.N^{2}\right)\right) H_{d}$, where

$$
\begin{aligned}
& N=\sqrt{\frac{Z_{01}}{Z_{02}}}, \\
& n=\frac{Z 0}{\sqrt{Z_{01} Z_{02}}}, \\
& n_{1}=\frac{Z_{0}}{Z_{01}}, \\
& n_{2}=\frac{Z_{0}}{Z_{02}},
\end{aligned}
$$

$H_{j}= \begin{cases}1 \text { on the sub domain } j, & j=\text { source, dielectric, metal. } \\ 0 \text { elsewhere, } & j=\text { source, dielectric, metal. }\end{cases}$

The incident waves are reflected on the upper and lower parts of the metallic box. So the relationship between waves $\left(A_{i}^{\alpha}\right.$ and $B_{i}^{\alpha}(\alpha=$ TE, TM $\left.)\right)$ in spectral domain is $[1,3]$

$$
\left[\begin{array}{c}
B_{i}^{\mathrm{TE}} \\
B_{i}^{\mathrm{TM}}
\end{array}\right]=\left[\begin{array}{cc}
\Gamma_{i}^{\mathrm{TE}} & 0 \\
0 & \Gamma_{i}^{\mathrm{TM}}
\end{array}\right]\left[\begin{array}{c}
A_{i}^{\mathrm{TE}} \\
A_{i}^{\mathrm{TM}}
\end{array}\right]
$$

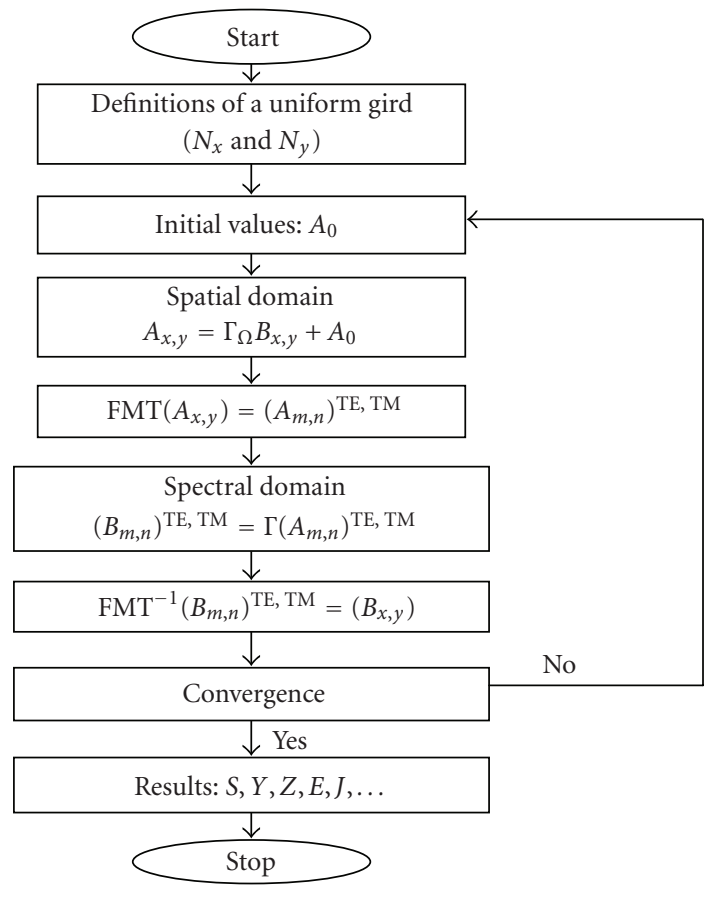

FIGURE 2: Iterative processes algorithm.

where

$$
\begin{aligned}
\Gamma_{i}^{\alpha} & =\frac{1-Z_{0 i} Y_{m n, i}^{\alpha} \operatorname{Coth}\left(\gamma_{m n, i} h_{i}\right)}{1+Z_{0 i} Y_{m n, i}^{\alpha} \operatorname{Coth}\left(\gamma_{m n, i} h_{i}\right)}, \\
Y_{m n, i}^{\mathrm{TE}} & =\frac{\gamma_{m n, i}}{j \omega \varepsilon_{0} \mu_{0}}, \\
Y_{m n, i}^{\mathrm{TM}} & =\frac{j \omega \varepsilon_{0} \mu_{0}}{\gamma_{m n, i}}, \\
\gamma_{m n, i} & =\sqrt{\left(\frac{m \pi}{a}\right)^{2}+\left(\frac{n \pi}{b}\right)^{2}-k_{0}^{2} \varepsilon_{r i} .}
\end{aligned}
$$

$h_{i}$ : the $I$ middle height.

This approach could then be considered as alternative between spatial and spectral domains. From the passage 


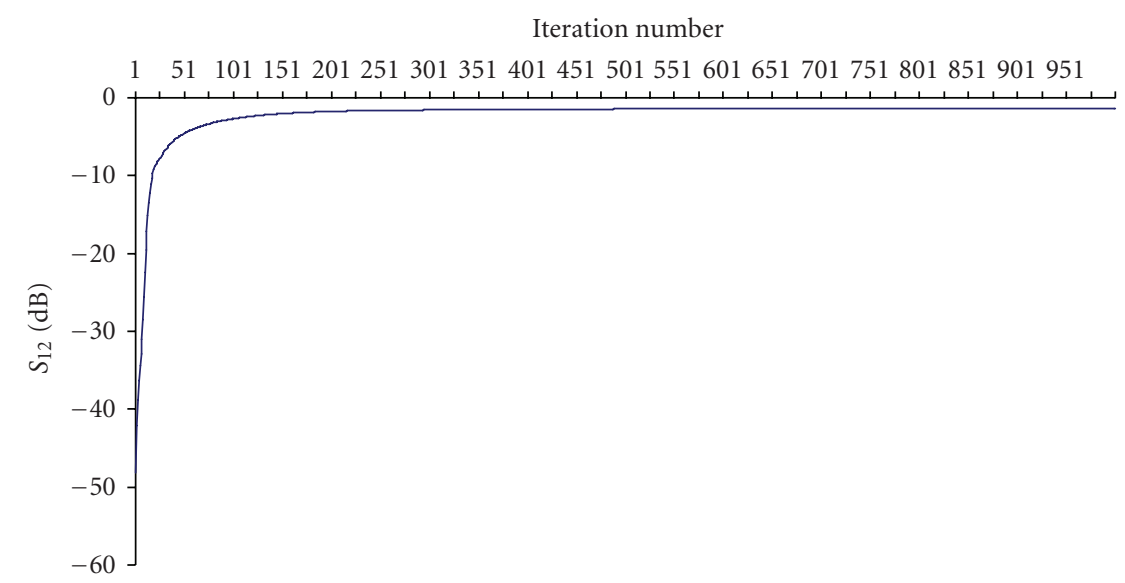

FIGURE 3: $S_{12}$ versus iterations number.

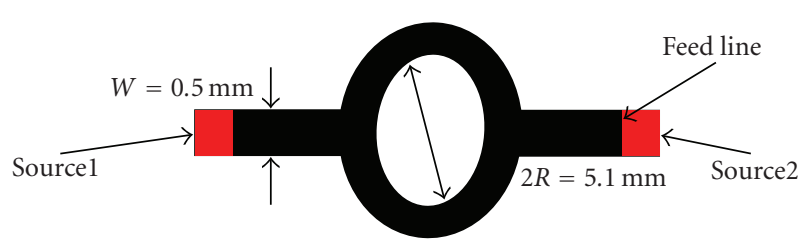

FIgURE 4: Ring resonator geometry.

spatial to spectral domain, we use a 2D-FMT algorithm in wave guide environment (see Appendix B).

The iterative process continues to the nth iteration, when the electric field and the current density converge; the iterative process is stopped. It is possible to calculate $E_{i}$ and $J_{i}$ at the interface $\Omega$ :

$$
\begin{aligned}
E_{i} & =\sqrt{Z_{o i}}\left(A_{i}+B_{i}\right), \\
J_{i} & =\frac{1}{\sqrt{Z_{o i}}}\left(A_{i}-B_{i}\right) .
\end{aligned}
$$

Finally, it is possible to determine the admittance matrix, from that, the scattering matrix $S_{i j}$ between the two ports can be obtained by the following equation:

$$
[S]=[1-[Y]][1+[Y]]^{-1}
$$

$[Y]$ is the admittance matrix.

The algorithm of the iterative process is given by Figure 2 . $N_{x}$ and $N_{y}$ : the numbers of pixels in the plan $\Omega$.

$A_{m, n}$ and $B_{m, n}$ : incident wave and reflected wave, respectively, in the spectral domain.

$A_{x, y}$ and $B_{x, y}$ : incident wave and reflected wave, respectively, in the spatial domain.

Figure 3 shows the $S_{12}$ convergence at $5 \mathrm{GHz}$ for the structure given by Figure 4 .

We can see that the iterative process converges at 350 iterations. So we can calculate $S_{12}$ versus frequency, using at each frequency the same number of the iterations.

\section{Results and Illustrations}

Figure 5 shows the simulated and measured spectra of transmitted microwave $\left(S_{12}\right)$ of the microstip ring with the directly connected feedline.

The geometry and dimensions of the regular ring are given by Figure 4; it is fabricated on a RT/Duriod 6010 substrate.

A comparison between the simulation results and measured results [4] is done; a good agreement is observed. The mismatch between the simulation and measurement data is inferior to $6 \mathrm{~dB}$ for the majority of frequencies. We note that the regular ring shows $S_{12}$ parameter similar to those of conventional transmission line.

The introduction of a small gap in the closed loop of the regular ring induces strong attenuations around $6.5 \mathrm{GHz}$ and $20 \mathrm{GHz}$ corresponding to $n=1$ and $n=3$ in (9):

$$
f_{n}=\frac{n c}{2 \pi R \sqrt{\varepsilon_{\text {eff }}}}
$$

where $2 \pi R=n \lambda$ and $\varepsilon_{\text {eff }}=6.77$ are given by simulation by [4], $c$ is speed of light and $\lambda$ is wavelength.

The new geometry of the ring is given by Figure 6; the dimension of the gap is $g=0.2 \mathrm{~mm}$. This structure induces multiple reflections with a fixed phase correlation.

The measured and simulated reflected coefficients $\left(S_{11}\right)$ are illustrated in Figure 7, and Figure 8 shows the transmitted coefficient $\left(S_{21}\right)$. We note the absence of the stop band around $13 \mathrm{GHz}$ correspond to $n=2$ in (9), because the microwave propagation along the ring is successively reflected with the fixed phase correlation when they satisfy $2 \pi R-G=n \lambda$, where $G$ is the gap dimension.

We see a satisfactory argument between, respectively, measured [4] and simulated reflected coefficient $S_{11}$ and transmitted coefficient $S_{12}$.

In the frequency range from $12 \mathrm{GHz}$ to $17 \mathrm{GHz}$, the measurement data for $\left(S_{11}\right)$ show distinct standing wave behavior. This does not have any physical interest. They are disturbances because of the complexity of the structure.

Because a ring resonator can be represented by a parallel LC circuit of inductance $L$ and capacitance $C$, introduction 


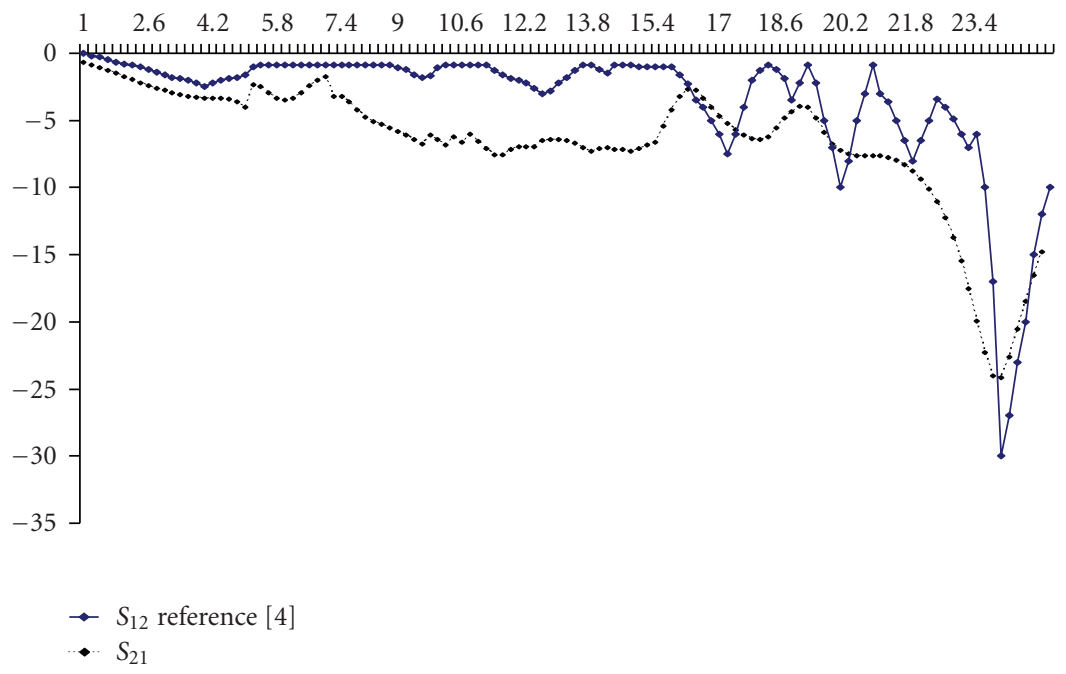

FIgURE 5: Transmission coefficient of the ring resonator.

of a narrow gap in the ring slightly changes the values of $C$ and $L$ from the original ones. The value of $C$ depends on the gap width $G$, the line width $w$, and the circumference $2 \pi R$, while the value of $L$ depends on the value of $w$ and $2 \pi R$.

Thus the attenuations valleys around $6.5 \mathrm{GHz}$ and $20 \mathrm{GHz}$, witch were explained to be due to multiple reflections occurring at the gap, correspond to the resonance frequencies of the equivalent LC circuit of the ring.

If we change the values of $G$, we can change the capacitance $C$, consequently the resonance frequency corresponding to $\omega=1 / \sqrt{L C}$ takes another values.

Figure 9 shows the simulated transmitted coefficient $\left(S_{12}\right)$ of different values of $G$; we can observe the shift of the two attenuations valleys.

Another method can be used for varying the capacitance $C$; we can mount a varactor diode at the gap of the ring (see Figure 10), when a varactor is connected parallel to the gap, the total capacitance at the discontinuity is increased to the sum of the varactor capacitance and the gap capacitance.

In simulation, the varactor diode is considered an auxiliary source that is used to study in a disjoined way two distinct parts of a circuit [5].

The expression of the density of current according to electric field for the two principal's source and the auxiliary source will be according to following equations:

$$
\begin{aligned}
& J_{1}=Y_{11} E_{1}+Y_{12} E_{2}+Y_{13} E_{3}, \\
& J_{2}=Y_{21} E_{1}+Y_{22} E_{2}+Y_{23} E_{3}, \\
& J_{3}=Y_{31} E_{1}+Y_{32} E_{2}+Y_{33} E_{3} .
\end{aligned}
$$

$\left(J_{1}, E_{1}\right)$ and $\left(J_{2}, E_{2}\right)$ : density of current and electric field in the tow principal's source.

$\left(J_{3}, E_{3}\right)$ : density of current and electric field in the auxiliary source.

$Y_{i j}$ are the coupling admittance between different sources determinate with the iterative method.

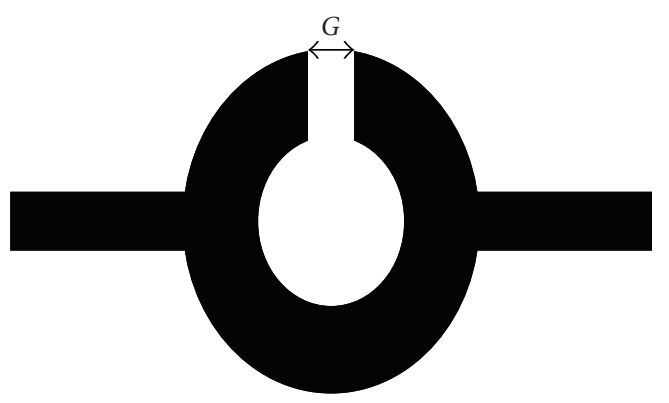

FIgURE 6: Ring with gap.

Figure 11 shows the equivalent circuit of the varactor diode.

According to this circuit, we can deduce the following relation:

$$
I_{D}=\left(\frac{j \omega C_{j}(0)}{j \omega C_{j}\left(R_{s}+j \omega L_{p}\right)+\sqrt{1-\left(V_{D} / \phi\right)}}+j \omega C_{p}\right) V_{D}
$$

With $V L=E_{3} G$ and $I_{L}=J_{3} W, W$ and $G$ are the diode dimensions.

If $W=G$, we obtained

$$
J_{3}=\left(\frac{j \omega C_{j}(0)}{j \omega C_{j}\left(R_{s}+j \omega L_{p}\right)+\sqrt{1-E_{3} / \phi}}+j \omega C_{p}\right) E_{3} .
$$

The value of $E_{3} / \phi$ tends towards zero, therefore we will have

$$
J_{3}=\left(\frac{j \omega C_{j}(0)}{j \omega C_{j}\left(R_{s}+j \omega L_{p}\right)+\left(1-E_{3} / 2 \phi\right)}+j \omega C_{p}\right) E_{3} .
$$




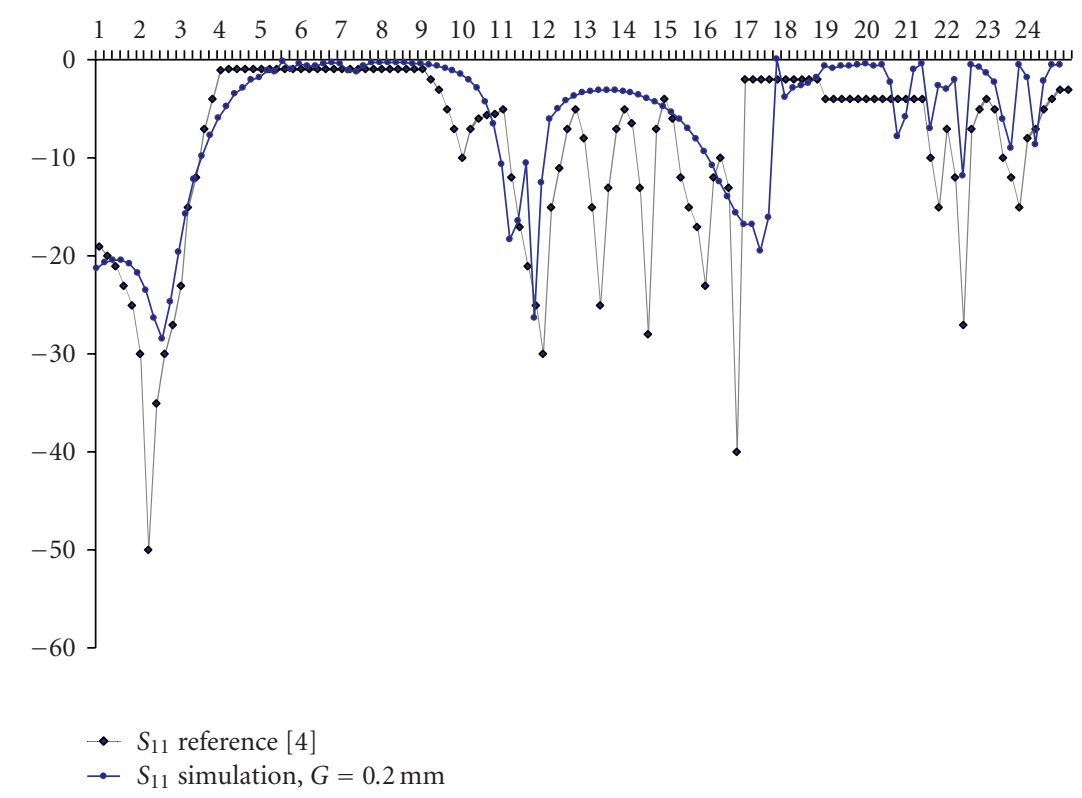

FIGURE 7: Reflection coefficient $S_{11}$ of the microstrip ring with a narrow gap.

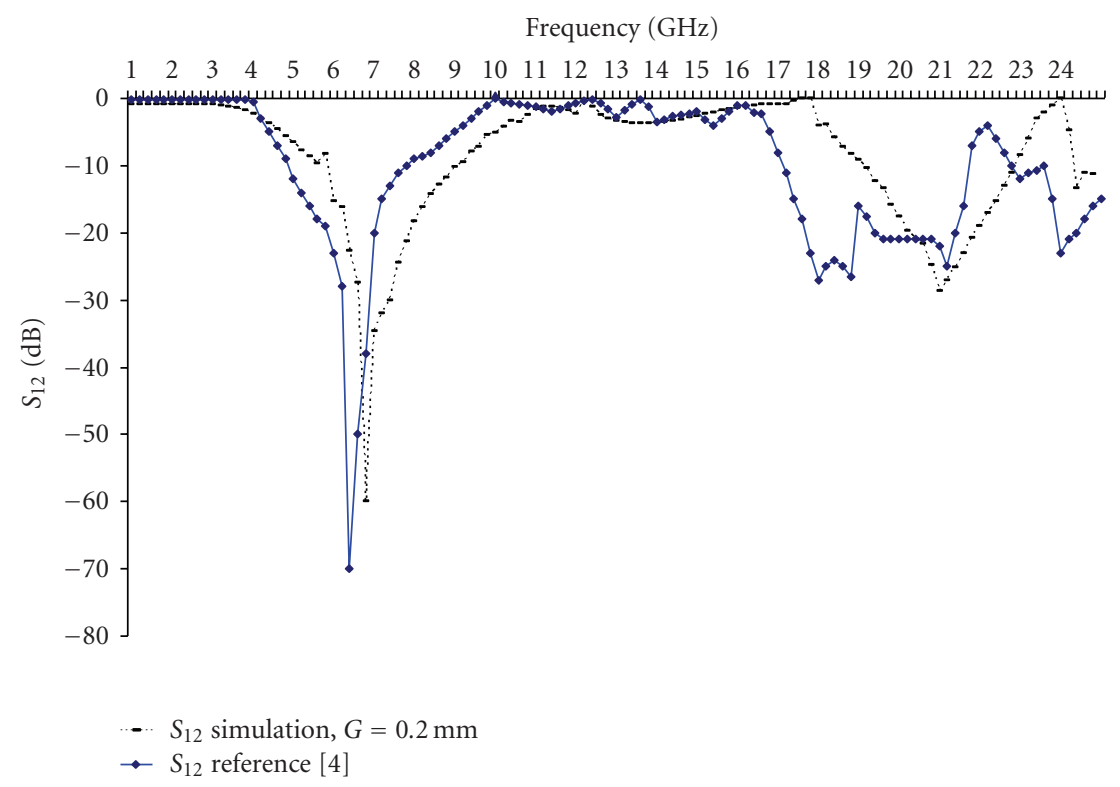

FIgURE 8: Transmission coefficient $S_{12}$ of the ring with a narrow gap.

Using (13) and (10b), we have

$$
\begin{aligned}
Y_{31} E_{1} & +Y_{32} E_{2} \\
& =\left(j \omega C_{p}-Y_{33}\right) E_{3}+\frac{j \omega C_{j} E_{3}}{j \omega C_{j}\left(R_{s}+j \omega L_{p}\right)+\left(1-E_{3} / 2 \phi\right)} .
\end{aligned}
$$

Well,

$$
\frac{j \omega C_{j} E_{3}}{j \omega C_{j}\left(R_{s}+j \omega L_{p}\right)+\left(1-E_{3} / 2 \phi\right)} \approx \frac{j \omega C_{j} E_{3}}{j \omega C_{j}\left(R_{s}+j \omega L_{p}\right)-1} .
$$

Consequently, (14) becomes

$$
\begin{aligned}
Y_{31} E_{1} & +Y_{32} E_{2} \\
& =\left(j \omega C_{p}-Y_{33}+\frac{j \omega C_{j}}{j \omega C_{j}\left(R_{s}+j \omega L_{p}\right)-1}\right) E_{3} \\
& \Longrightarrow E_{3}=\frac{Y_{31} E_{1}+Y_{32} E_{2}}{j \omega C_{p}-Y_{33}+j \omega C_{j} /\left(j \omega C_{j}\left(R_{s}+j \omega L_{p}\right)-1\right)} .
\end{aligned}
$$

Replacing $E_{3}$ in (10a) and (10b), we obtain

$$
\begin{aligned}
& J_{1}=\left(Y_{11}+Y_{13} K_{1}\right) E_{1}+\left(Y_{12}+Y_{13} K_{2}\right) E_{2}, \\
& J_{2}=\left(Y_{21}+Y_{23} K_{1}\right) E_{1}+\left(Y_{22}+Y_{23} K_{2}\right) E_{2} .
\end{aligned}
$$




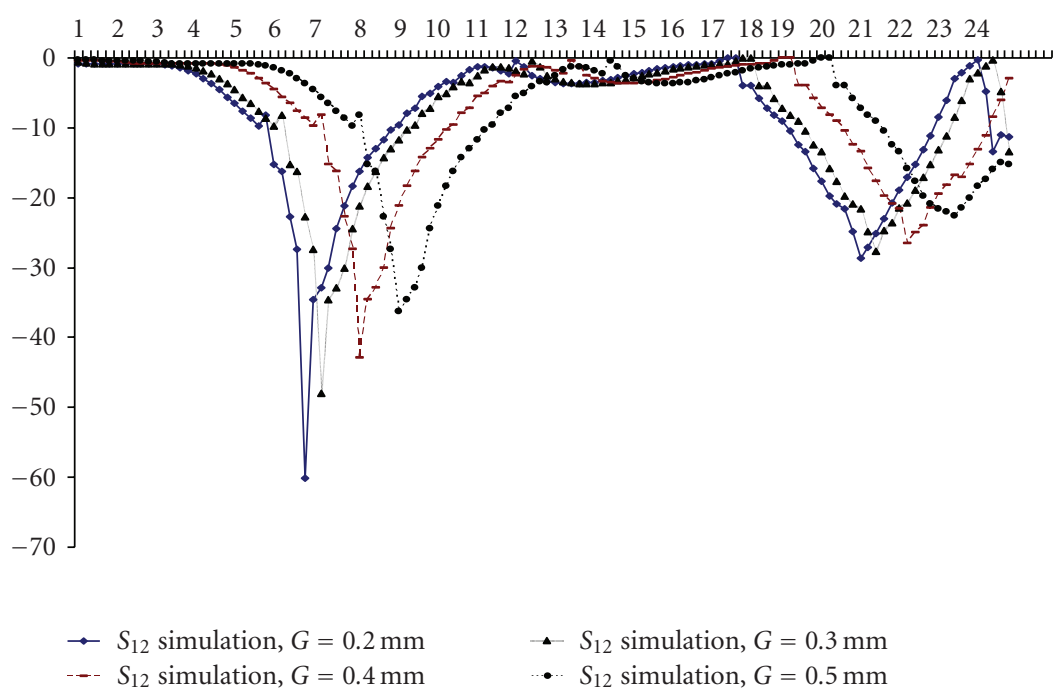

FIgURE 9: Transmission characteristics of the ring resonator for different value of $G$.

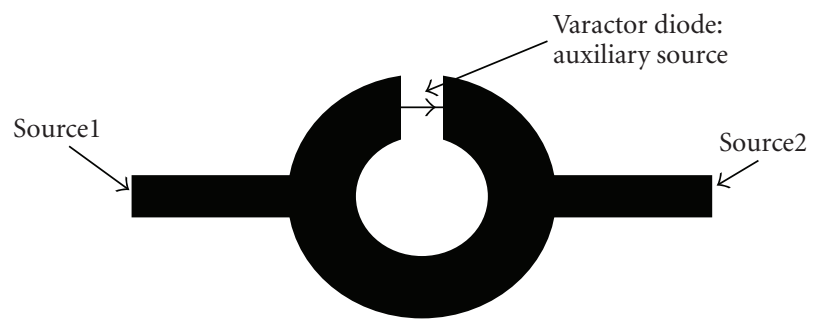

Figure 10: Ring with varactor diode.

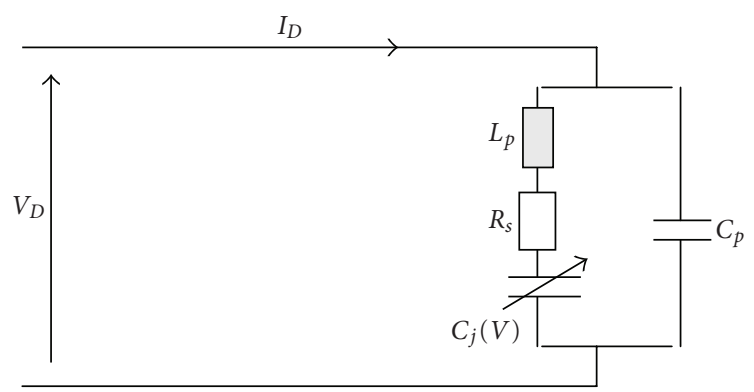

Figure 11: Equivalent circuit of the varactor diode.

With

$$
\begin{aligned}
K_{1} & =\frac{Y_{31}}{j \omega C_{p}-Y_{33}+j \omega C_{j} /\left(j \omega C_{j}\left(R_{s}+j \omega L_{p}\right)-1\right)}, \\
K_{2} & =\frac{Y_{32}}{j \omega C_{p}-Y_{33}+j \omega C_{j} /\left(j \omega C_{j}\left(R_{s}+j \omega L_{p}\right)-1\right)} .
\end{aligned}
$$

The admittance matrix of the ring with varactor diode is given by (19)

$$
Y=\left[\begin{array}{ll}
Y_{11}+Y_{13} K_{1} & Y_{12}+Y_{13} K_{2} \\
Y_{21}+Y_{23} K_{1} & Y_{22}+Y_{23} K_{2}
\end{array}\right]
$$

The simulated results for the transmission coefficient of ring with a narrow gap and the ring with the varactor mounted at the gap are shown in Figure 12; we can see the new valley obtained with the varactor.

\section{Conclusion}

In this paper, we have proposed a microstrip ring that exhibits PBG characteristics. The stop band of the ring resonator is caused by multiple reflections with a fixed phase correlation at the gap; we saw different technique for the control of those stopped bands; the results are determined by using an iterative technique. The comparison of numerical results with the measurement published data verified the validation of the proposed technique. This technique takes the advantage of simplicity and it is in conjunction with the 2D-FMT which allows a high computational speed and memory consumption.

\section{Appendices}

\section{A. Amplitude of the mode in metallic box}

The metallic box modes are developed as the following equations:

$$
\begin{aligned}
e^{\mathrm{TE}}(m, n) & =\sum_{i, j} a^{\mathrm{TE}}(i, j)\left|f^{\mathrm{TE}}(i, j)\right\rangle, \\
e^{\mathrm{TM}}(m, n) & =\sum_{i, j} a^{\mathrm{TM}}(i, j)\left|f^{\mathrm{TM}}(i, j)\right\rangle .
\end{aligned}
$$

The magnitude function is written as

$$
\begin{aligned}
{\left[\begin{array}{l}
a^{\mathrm{TE}} \\
a^{\mathrm{TM}}
\end{array}\right] } & =[T]\left[\begin{array}{l}
e_{x} \\
e_{y}
\end{array}\right], \\
{[T] } & =\kappa\left[\begin{array}{cc}
-\frac{n}{b} & \frac{m}{a} \\
\frac{m}{a} & \frac{n}{b}
\end{array}\right] .
\end{aligned}
$$




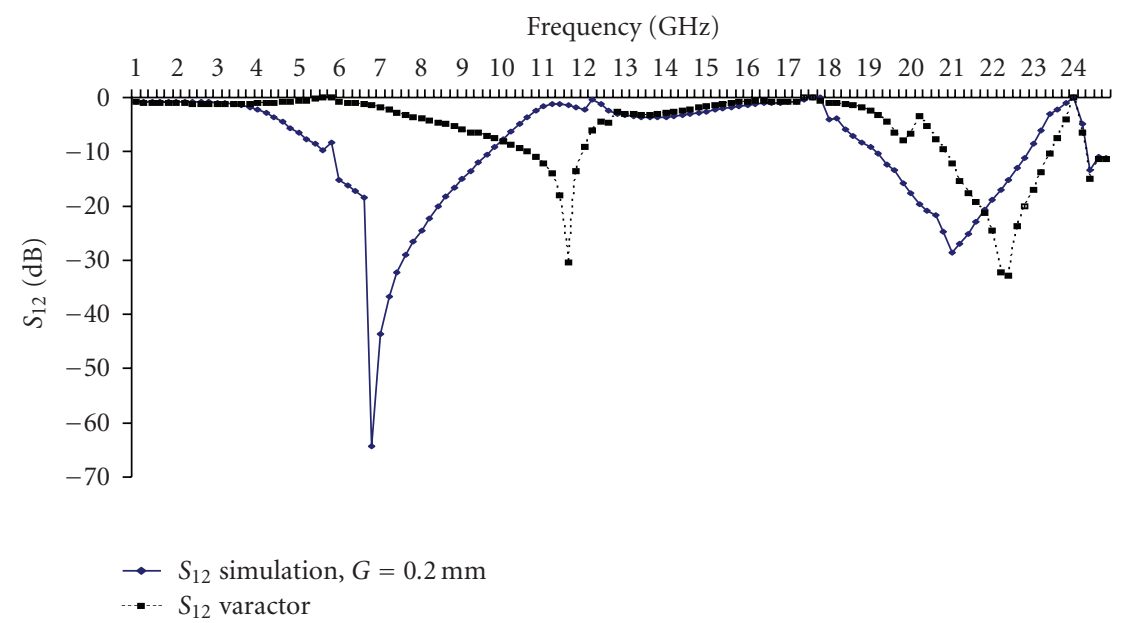

FIgURE 12: Transmission coefficient with and without varactor diode.

With

$$
\begin{aligned}
\kappa & =\frac{1}{\sqrt{(m / A)^{2}+(n / B)^{2}}} \sqrt{2 \sigma_{m n}} / a b, \\
\sigma_{m n} & = \begin{cases}1 & \text { if } \min =0 \\
2 & \text { if } \min \neq 0 .\end{cases}
\end{aligned}
$$

Using $A_{1}$ and $A_{2}$, it is possible to establish

$$
\begin{array}{r}
e^{\mathrm{TE}}(m, n) \\
=\sum_{i, j} \frac{n}{b} e_{x}(i, j)\left|f_{x}(i, j)\right\rangle+\sum_{i, j} \frac{m}{a} e_{y}(i, j)\left|f_{y}(i, j)\right\rangle, \\
e^{\mathrm{TM}}(m, n) \\
=\sum_{i, j} \frac{n}{b} e_{y}(i, j)\left|f_{y}(i, j)\right\rangle+\sum_{i, j} \frac{m}{a} e_{x}(i, j)\left|f_{x}(i, j)\right\rangle .
\end{array}
$$

The base function for metallic box is given by

$$
\begin{aligned}
f_{x} & =\cos \left(\frac{m \pi i}{M}\right) \sin \left(\frac{n \pi j}{N}\right), \\
f_{y} & =\sin \left(\frac{m \pi i}{M}\right) \cos \left(\frac{n \pi j}{N}\right), \\
\frac{i}{M} & =\frac{x}{a}, \\
\frac{j}{N} & =\frac{y}{b} .
\end{aligned}
$$

Moreover, $\left(A_{3}\right)$ becomes

$$
\begin{aligned}
e^{\mathrm{TE}}(m, n)= & \sum_{i, j} \frac{n}{b} e_{x}(i, j) \cos \left(\frac{m \pi i}{M}\right) \sin \left(\frac{n \pi j}{N}\right) \\
& +\sum_{i, j} \frac{n}{b} e_{y}(i, j) \sin \left(\frac{m \pi i}{M}\right) \cos \left(\frac{n \pi j}{N}\right),
\end{aligned}
$$

$$
\begin{aligned}
e^{\mathrm{TM}}(m, n)= & \sum_{i, j} \frac{n}{b} e_{z}(i, j) \sin \left(\frac{m \pi i}{M}\right) \cos \left(\frac{n \pi j}{N}\right) \\
& +\sum_{i, j} \frac{n}{b} e_{x}(i, j) \cos \left(\frac{m \pi i}{M}\right) \sin \left(\frac{n \pi j}{N}\right) .
\end{aligned}
$$

\section{B. Fast Modal Transform FMT}

From the passage spatial to spectral domain in a rectangular wave guide environment, we use a 2D-FMT (TowDimensions Fast Modal Transform), deduce from the twodimensions Fourier transform.

The two-dimensions usual Fourier transform of the function $E(x, y)$ can be written by the following equation:

$$
e^{\prime}(m, n)=\sum_{i, j}^{2 M, 2 N} E_{x, y} e^{-2 j\left(\pi m x_{i} / A\right)} e^{-2 j\left(\pi n y_{i} / B\right)} .
$$

According to $x, E\left(x_{i}, y_{j}\right)$ is an odd function, so $E\left(x_{i}, y_{j}\right)=$ $-E\left(2 A-x_{i}, y_{j}\right)$,

$$
\begin{aligned}
e^{\prime}(m, n)= & \sum_{i, j}^{M, 2 N} E_{x, y}\left(e^{-2 j\left(\pi m x_{i} / A\right)}-e^{-4 j \pi m} e^{2 j\left(\pi m x_{i} / A\right)}\right) e^{-2 j\left(\pi n y_{j} / B\right)}, \\
e^{\prime}(m, n)= & \sum_{i, j}^{M, 2 N} E_{x, y} e^{-2 j \pi m} \\
& \times\left(e^{2 j \pi m} e^{-2 j\left(\pi m x_{i} / A\right)}-e^{-2 j \pi m} e^{2 j\left(\pi m x_{i} / A\right)}\right) e^{-2 j\left(\pi n y_{j} / B\right)}, \\
e^{\prime}(m, n)= & \sum_{i, j}^{M, 2 N} E_{x, y} e^{-2 j \pi m} \\
& \times\left(e^{-2 j\left(\pi m x_{i} / A-\pi m\right)}-e^{2 j\left(\pi\left(\pi m x_{i} / A\right)-\pi m\right)}\right) e^{-2 j\left(\pi n y_{j} / B\right)},
\end{aligned}
$$




$$
\begin{aligned}
e^{\prime}(m, n)= & \sum_{i, j}^{M, 2 N} E_{x, y} \\
& \times e^{-2 j \pi m}\left(e^{-2 j\left(\pi m x_{i} / A\right)}-e^{2 j\left(\pi\left(\pi m x_{i} / A\right)\right)}\right) e^{-2 j\left(\pi n y_{j} / B\right)}, \\
e^{\prime}(m, n)= & -2 j \sum_{i, j}^{M, 2 N} E_{x, y} e^{-2 j \pi m} \sin \left(\frac{2 \pi m x_{i}}{A}\right) e^{-2 j\left(\pi n y_{j} / B\right)} .
\end{aligned}
$$

Of the other share $E\left(x_{i}, y_{j}\right)$ is an even function according to $y$, so, $E\left(x_{i}, y_{j}\right)=E\left(x_{i}, 2 B-y_{j}\right)$

$$
\begin{aligned}
e^{\prime}(m, n)= & -2 j \sum_{i, j}^{M, N} E_{x, y} e^{-2 j \pi m} \sin \left(\frac{\pi m x_{i}}{A}\right) \\
& \times\left(e^{-2 j\left(\pi n y_{j} / B\right)} e^{-4 j \pi n}+e^{2 j\left(\pi n y_{j} / B\right)}\right), \\
e^{\prime}(m, n)= & -2 j \sum_{i, j}^{M, N} E_{x, y} e^{-2 j \pi m} \sin \left(\frac{\pi m x_{i}}{A}\right) e^{-2 j \pi n} \\
& \times\left(e^{-2 j \pi n} e^{-2 j\left(\pi n y_{j} / B\right)}+e^{2 j \pi n} e^{2 j\left(\pi n y_{j} / B\right)}\right), \\
e^{\prime}(m, n)= & -2 j \sum_{i, j}^{M, N} E_{x, y} e^{-2 j \pi m} \sin \left(\frac{\pi m x_{i}}{A}\right) e^{-2 j \pi n} \\
& \times\left(e^{-2 j\left(\pi n+\pi n y_{j} / B\right)}+e^{2 j\left(\pi n+\pi n y_{j} / B\right)}\right), \\
e^{\prime}(m, n)= & -2 j \sum_{i, j}^{M, N} E_{x, y} e^{-2 j \pi m} \sin \left(\frac{\pi m x_{i}}{A}\right) e^{-2 j \pi n} \\
& \times\left(e^{-2 j\left(\pi n y_{j} / B\right)}+e^{2 j\left(\pi n y_{j} / B\right)}\right), \\
& \times \sin \left(\frac{2 \pi m x_{i}}{A}\right) \cos \left(\frac{2 \pi n x_{j}}{B}\right) . \\
e^{\prime}(m, n)= & -4 j \sum_{i, j}^{M, N} E_{x, y} e^{-2 j \pi n} e^{-2 j \pi m}
\end{aligned}
$$

Consequently, we can write the FMT using the usual Fourier transform:

$$
e(m, n)=\frac{j}{4} e^{2 j \pi m} e^{2 j \pi n} e^{\prime}(m, n) .
$$

\section{References}

[1] A. Mami, H. Zairi, A. Gharsallah, and H. Baudrand, "Analysis of microstrip spiral inductor by using iterative method," Microwave and Optical Technology Letters, vol. 35, no. 4, pp. 302-306, 2002.

[2] A. Gharsallah, A. Gharbi, L. Desclos, and H. Baudrand, "Analysis of interdigital capacitor and quasi-lumped miniaturized filters using iterative method," International Journal of Numerical Modelling: Electronic Networks, Devices and Fields, vol. 15, no. 2, pp. 169-179, 2002.

[3] M. Yeddes, M. Kaddour, A. Gharsallah, and A. Gharbi, "Analysis of DGS structures by using the iterative method," International Journal of Numerical Modelling: Electronic Networks, Devices and Fields, vol. 18, no. 4, pp. 297-311, 2005.
[4] C.-S. Kee, M.-Y. Jang, I. Park, et al., "Photonic band gap formation by microstrip ring: a way to reduce the size of microwave photonic band gap structures," Applied Physics Letters, vol. 80, no. 9, pp. 1520-1522, 2002.

[5] M. Yeddes, H. Zairi, A. Gharsallah, A. Gharbi, and H. Baudrant, "A global electromagnetic modeling of an active microstrip structure," International Journal of $R F$ and Microwave Computer-Aided Engineering, vol. 17, no. 4, pp. 425-434, 2007. 

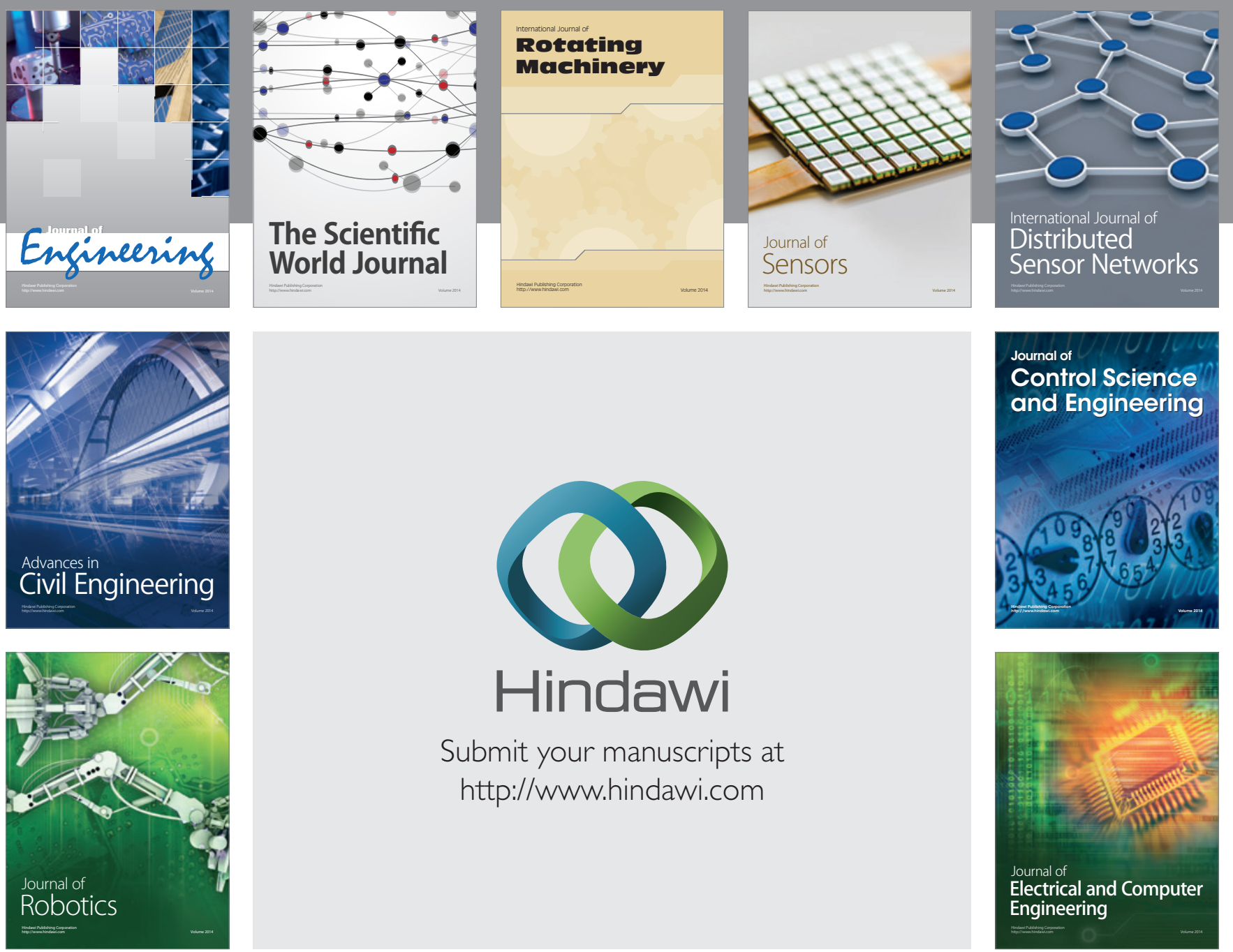

Submit your manuscripts at

http://www.hindawi.com
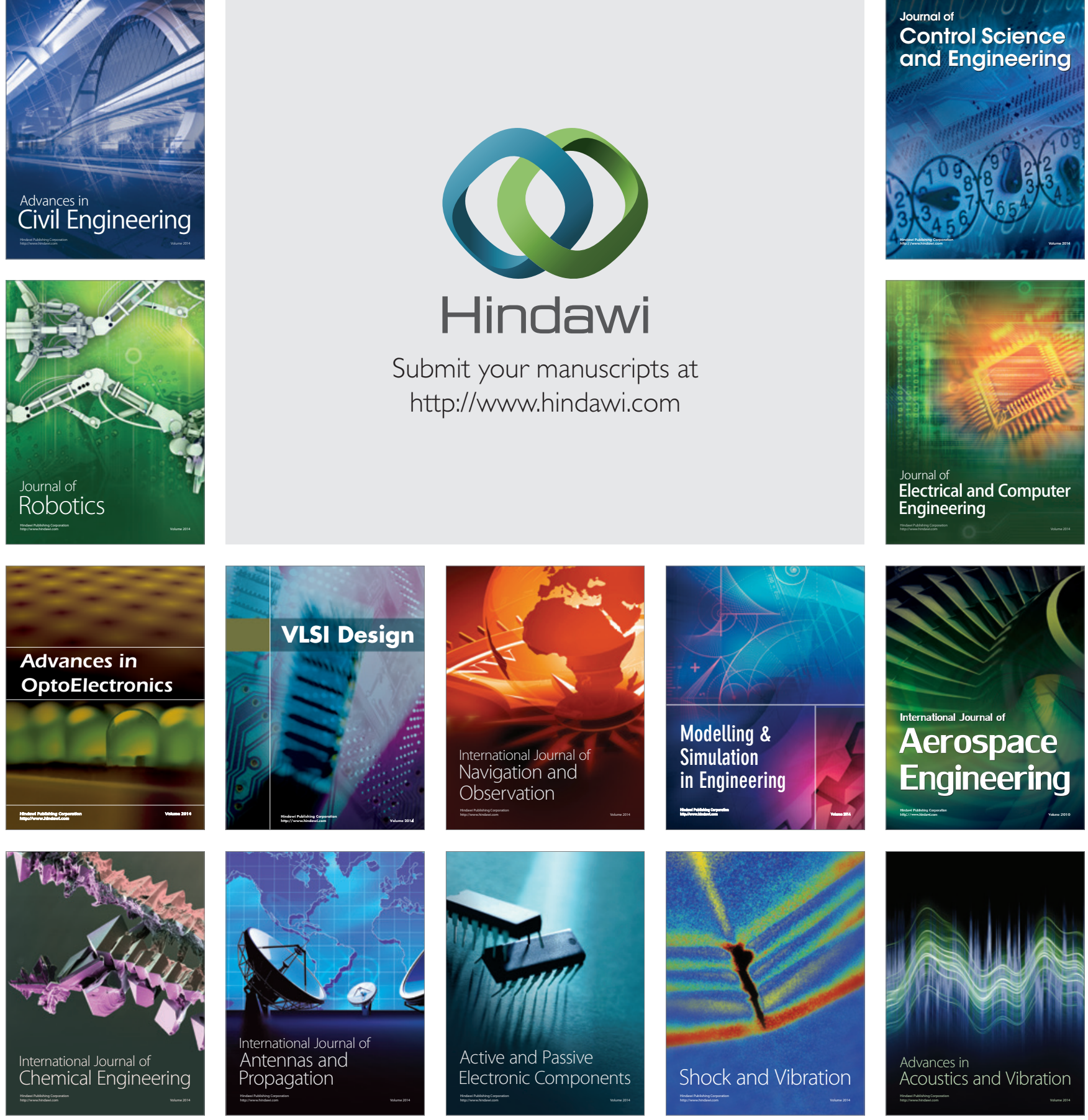